\title{
Lead-silicate glass optical microbubble resonator
}

\author{
Pengfei Wang, ${ }^{1,2, a, b)}$ Jonathan Ward, ${ }^{3, b)}$ Yong Yang, ${ }^{3, b)}$ Xian Feng, ${ }^{2, b)}$ Gilberto \\ Brambilla, ${ }^{2}$ Gerald Farrell ${ }^{1}$ and Síle Nic Chormaic ${ }^{3}$ \\ ${ }^{1}$ Photonics Research Centre, Dublin Institute of Technology, Kevin Street, Dublin 8, Ireland \\ ${ }^{2}$ Optoelectronics Research Centre, University of Southampton, Southampton SO17 1BJ, United Kingdom \\ ${ }^{3}$ Light-Matter Interactions Unit, OIST Graduate University, 1919-1 Tancha, Onna-son, Okinawa 904-0495, Japan
}

\begin{abstract}
Microbubble whispering gallery resonators have the potential to become key components in a variety of active and passive photonic circuit devices by offering a range of significant functionalities. Here, we report on the fabrication, optical characterization, and theoretical analysis of lead-silicate glass, optical microbubble resonators. Evanescent field coupling to the microbubbles was achieved using a $1 \mu \mathrm{m}$ diameter, silica microfiber at a wavelength of circa $775 \mathrm{~nm}$. High Q-factor modes were efficiently excited in both single-stem and two-stem, lead-silicate glass, microbubble resonators, with bubble diameters of $38 \mu \mathrm{m}$ (single-stem) and $48 \mu \mathrm{m}$ (two-stem). Whispering gallery mode resonances with Q-factors as high as $2.3 \times 10^{5}$ (single-stem) and $7 \times 10^{6}$ (two-stem) were observed. By exploiting the high-nonlinearity of the lead-silicate glass, this work will act as a catalyst for studying a range of nonlinear optical effects in microbubbles, such as Raman scattering and four-wave mixing, at low optical powers.
\end{abstract}

Over the past few decades whispering gallery mode resonators, such as microdisks, microrings, microspheres, and microtoroids have been investigated intensively across a wide range of applications, such as bio and chemical sensing [1-3], and in the study of fundamental physics, such as optical force trapping [4], optomechanics [5,6] and cavity quantum electrodynamics [7,8]. In 2010, a silica optical microbubble resonator was fabricated [9] by heating the mid-section of a pressurized rotating silica glass microcapillary with a $\mathrm{CO}_{2}$ laser. The microcapillary expanded under internal air pressure and a microbubble was formed at the midsection with a diameter of circa $300 \mu \mathrm{m}$ and a wall thickness of $\sim 2$ $\mu \mathrm{m}$. $\mathrm{A} \mathrm{CO}_{2}$ laser heating technique was used because it provides a reasonably stable radiative heat source regardless of the influence from external airflow compared with the more conventional heating

P. Wang to whom correspondence should be addressed. Electronic mail: pengfei.wang@dit.ie

P. Wang, J. Ward, Y. Yang and X. Feng contributed equally to this work. 
technique using a flame torch. The amount of heat required to keep the glass soft increases exponentially as the microbubble wall thickness decreases [10] or, conversely, for a focused $\mathrm{CO}_{2}$ laser power, the temperature of the bubble wall decreases as the absorbed power decreases. As a result, at some point during the expansion of the bubble, the convective heat loss from its surface is greater than the heating rate and the glass rapidly cools, thereby preventing any further expansion. In this way, the diameter and wall thickness of the bubble can be controlled using different internal air pressures and $\mathrm{CO}_{2}$ laser powers.

Recent research concerning all-optical frequency comb generation has mainly relied on a thirdorder, optical nonlinearity in a two-stem silica microbubble with a diameter of $136 \mu \mathrm{m}$ and a wall thickness of 3-4 $\mu \mathrm{m}$ [11]. Therefore, in order to explore other nonlinear properties it would be desirable to fabricate microbubble resonators from glasses with other material properties, e.g. compound glass materials with the unique properties of higher refractive indices (for tighter mode confinement) and larger optical nonlinearities. For example, lead-silicate $\left(\mathrm{SiO}_{2}-\mathrm{PbO}\right)$ glasses with a high content of leadoxide have a nonlinear refractive index $\left(n_{2}\right)$ that can be as high as 20 times that of conventional silica [12]. Glasses with high nonlinearity are of immense importance for devices performing optical switching and frequency comb generation, thus a high Q-factor lead-silicate glass microbubble resonator is promising for realizing such nonlinear processes with very low-threshold power. This should permit substantially reduced thresholds for nonlinear processes such as Raman scattering or four-wave mixing in optical resonators. Since compound glass, optical microbubble resonators have not yet been addressed, in this letter we report on the fabrication of such microbubbles and their optical characterization.

Two types of bubble resonators were fabricated in this work, one of which was a single-stem microbubble with submicron wall thickness, and the other was more robust and symmetrical with two 
stems. The single-stem microbubble had a diameter around $38 \mu \mathrm{m}$ with a wall thickness of circa $0.8 \mu \mathrm{m}$; the two-stem microbubble sample has a diameter around $48 \mu \mathrm{m}$ and the wall thickness ranges from an estimated 1.1 to $1.2 \mu \mathrm{m}$ based on the measured microcapillary and microbubble dimension as described in [13]. Optical whispering gallery mode spectra were observed and the Q-factors of both microbubbles were measured. First of all, a lead-silicate, microcapillary tube was fabricated from Schott SF57 glass, which has a refractive index of $\mathrm{n} \sim 1.826$ at a wavelength of $\lambda=780 \mathrm{~nm}$. The outer diameter (OD) was $836.3 \mu \mathrm{m}$ and the inner diameter was $603.5 \mu \mathrm{m}$, as shown in Fig. 1(a). The capillary was next tapered down to a uniform OD of $\sim 18.6 \mu \mathrm{m}$ using a ceramic microheater and the tip was gently heated in the focus of two counterpropagating $\mathrm{CO}_{2}$ beams. This resulted in one end of the capillary closing and the remaining open end was connected to an air pump, which pressurized the air in the tube as the tip closed. When the tip was sealed, the air pressure in the tube forced the soft glass tip to expand and create a single-stem microbubble as shown in Fig.1 (b). Since the lead-silicate material is extremely soft and fragile, the power of the $\mathrm{CO}_{2}$ laser and the internal air pressure were controlled very accurately. With sufficient heating, the expansion of the bubble speeds up as the glass wall becomes thinner. At some point, the expansion can accelerate rapidly because the air pressure overcomes the surface tension forces. When the microbubble walls become too thin, the expansion stops suddenly once the rate of heat losses from the microbubble surface exceeds the heating rate. If there is too much heating, the microbubble will continue to expand until it bursts; the remaining material is reheated and forms a solid sphere and a new bubble begins underneath the solid sphere. Heating the capillary this way removed the need to rotate the capillary and the pretapering stage removes any requirement for chemical etching. It is possible to produce microbubbles with diameters $<50 \mu \mathrm{m}$ using this technique. Also, because of the small initial capillary diameter, the bubble wall thickness automatically becomes submicron during expansion. The diameter and wall thickness can be controlled by the degree of 
tapering, the amount of heating, and the gas pressure. Fig. 1 (b) and (c) show images of the single-stem and two-stem microbubbles made in our experimental setup.

Using a $\sim 1 \mu \mathrm{m}$ diameter tapered silica optical fiber, laser light at $\lambda \sim 775 \mathrm{~nm}$ from a mode-hop-free, tunable laser was coupled into the microbubbles and WGMs were excited by scanning the laser over 80 $\mathrm{GHz}$ with a scan frequency of $5 \mathrm{~Hz}$. Images of the microbubbles coupled with the silica fiber tapers are shown in Fig. 2 (a) and (b). Both the taper fiber and microbubble setups were placed on nanomotion precision piezo ceramic motors and the entire measurement system was placed inside an enclosure to remove the external environment disturbances, such as airflow.

Figure 3 (a) and (b) show the resonance spectra over $80 \mathrm{GHz}$ of the microbubbles with diameters of $38 \mu \mathrm{m}$ and $48 \mu \mathrm{m}$, respectively. The Q-factors of the microbubble resonators can be easily estimated from their WGM spectra and were $Q=2.3 \times 10^{5}$ ( ingle-stem sample) and $\mathrm{Q}=7 \times 10^{6}$ (two-stem sample). Note that the Q-factor of the single-stem sample is significantly smaller than that of the two-stem sample; when the microbubble wall thickness is thinner, the modes propagate to a greater extent within the core and this increases the radiation loss and reduces the Q-factor. On the other hand, similar to solid microsphere resonators, Q-factors increase with diameter. From the figures one can also see that the tapered fiber excitation produces dense spectral features as evidenced in other high index glass microspheres $[12,14]$. This is due to the excitation of many higher-order radial modes coupled by the low effective index silica fiber taper and many non-degenerate higher-order angular modes associated with microbubble ellipticity. The theoretical analysis with regard to this phenomenon is shown below.

Using a radio frequency (RF) module of the commercial Comsol Multiphysics software, Fig. 4 shows the results of the intensity patterns of WGMs in a microbubble with a diameter of $48 \mu \mathrm{m}$ and a wall thickness of $1.2 \mu \mathrm{m}$, where $\lambda=775 \mathrm{~nm}$, by a modified finite element method [15]. The radial order of a WGM is characterized by the integer number, $n$, where the number of intensity maxima in the radial 
direction is given by $n=1,2,3, \ldots$. The $n=1$ mode is confined mostly within the soft glass wall of the microbubble; however, as $n$ is increased more of the mode is distributed within the air core and thus reduces the effective index of the mode.

Next, the effective indices of the first three radial modes $(n=1-3)$ for a lead-silicate microbubble of $48 \mu \mathrm{m}$ diameter at $\lambda=775 \mathrm{~nm}$ were calculated for a wall thickness varying from 500 to $1500 \mathrm{~nm}$. The effective index of the fundamental mode in a $1 \mu \mathrm{m}$ tapered fiber is approximately 1.363 at $\lambda=775 \mathrm{~nm}$. A silica tapered fiber was used as a coupling waveguide to excite the microbubble WGMs instead of a phase-matched tapered high-index fiber so as to minimize any nonlinear effects from the delivery fiber. The simulation results shown in Fig. 5 indicate that the excited quasi-periodical WGM modes demonstrated in Fig. 3(b) were from the $n \geq 3$ high order modes. Also, since the power coupled into the WGM falls exponentially with the square of the phase mismatch, higher order radial modes, (and also higher order Hermite-Gaussian modes with $m<l$ ) which have $n_{\text {eff }}$ close to that of the microfiber mode, can be excited more efficiently than the fundamental WGM (when $n=1$ ). Furthermore, the resonance spectrum is complicated by the non-degeneracy of the aforementioned Hermite-Gaussian modes with different order $m$ due to the ellipticity observed in microbubbles. Fundamental modes with large $m$ have a reduced effective refractive index, thus can be effectively phase matched and efficiently excited. However, in practice, it is unlikely that the observed modes are fundamental high- $m$ order modes. High$m$ modes have a large longitudinal spatial distribution and in the real microbubbles used in our experiment will be quite lossy due to the existence of at least one or two thick stems attached to the microbubble, which scatter the light. These are not seen in the spectrum, as the modes still show high Q values.

In conclusion, we have shown that by first tapering a custom-made lead-silicate glass microcapillary tube, the size of WGM microbubbles can be reduced below $50 \mu \mathrm{m}$, thus allowing for the formation of 
optical microbubbles with both single-stem and two-stem geometrical structure and submicron wall thicknesses. Whispering gallery mode resonances with Q-factors as high as $2.3 \times 10^{5}$ (single-stem) and $7 \times 10^{6}$ (two-stem) were observed. With further improvements, it should be possible to make smaller diameter spherical bubbles with even thinner walls and higher optical Q-factors. These devices have many interesting optical effects. For example, by controlling the wall thickness, it is possible to modify the dispersion properties and also the nonlinearities of the microbubble resonators, thereby facilitating the observation of Raman scattering and four-wave mixing [11], etc. In terms of high nonlinearities of the lead-silicate glass material, further investigations of Raman scattering and four-wave mixing of the lead-silicate optical microbubbles are underway.

P. Wang is funded by the Royal Irish Academy Mobility Fellowship and the Irish Research Council “New Foundations 2013”. G. Brambilla gratefully acknowledges the Royal Society (London) for his university research fellowship. This publication has emanated from activity supported in part by Science Foundation Ireland (SFI) under the International Strategic Cooperation Award Grant Number SFI/13/ISCA/2845. This work was partially funded by OIST Graduate University. 


\section{References:}

[1]. F. Vollmer and S. Arnold, "Whispering-gallery-mode biosensing: label-free detection down to single molecules," Nature Methods 5, 591-596 (2008).

[2]. M. S. Luchansky, R. C. Bailey, "High-Q Optical Sensors for Chemical and Biological Analysis," Analytical Chemistry, 84, 793-821 (2012).

[3]. M. D. Baaske, M. R. Foreman and F. Vollmer "Single-molecule nucleic acid interactions monitored on a label-free microcavity biosensor platform," Nature Nanotechnology 180 (2014).

[4]. J. M. Ward, Y. Wu, V. G. Minogin, and S. Nic Chormaic, "Trapping of a microsphere pendulum resonator in an optical potential,” Phys. Rev. A 79, 053839 (2009).

[5]. T. J. Kippenberg and K. J. Vahala, "Cavity Optomechanics: Back-Action at the Mesoscale," Science 321, 1172-1176 (2008).

[6]. I. Favero and K. Karrai, “Optomechanics of deformable optical cavities," Nature Photon. 3, 201 (2009).

[7]. H. Mabuchi and A. C. Doherty, "Cavity Quantum Electrodynamics: Coherence in Context," Science 298, 1372-1377 (2002).

[8]. J. T. Shen and S. Fan, "Coherent Single Photon Transport in a One-Dimensional Waveguide Coupled with Superconducting Quantum Bits,” Phys. Rev. Lett. 95, 213001 (2005).

[9]. M. Sumetsky, Y. Dulashko, and R. S. Windeler, “Optical microbubble resonator,” Opt. Lett., 35, 898-900 (2010).

[10]. A. Watkins, J. Ward, Y. Wu and S. Nic Chormaic, "Single-input spherical microbubble resonator," Opt. Lett., 36, 2113-2115 (2011). 
[11]. M. Li, X. Wu, L. Liu, and L. Xu, "Kerr parametric oscillations and frequency comb generation from dispersion compensated silica micro-bubble resonators," Opt. Express 21, 16908-16913 (2013).

[12]. P. Wang, G. S. Murugan, T. Lee, X. Feng, Y. Semenova, Q. Wu, W. Loh, G. Brambilla, J. S. Wilkinson and G. Farrell, "Lead-silicate glass microsphere resonators with absorptionlimited Q," Applied Physics Letters, 98, 181105 (2011).

[13]. R. Henze, T. Seifert, J. Ward, and O. Benson, "Tuning whispering gallery modes using internal aerostatic pressure," Opt. Lett. 36, 4536-4538 (2011).

[14]. P. Wang, G. S. Murugan, G. Brambilla, T. Lee, M. Ding, Y. Semenova, Q. Wu, G. Farrell, "High-Q bismuth silicate nonlinear glass microsphere resonators," IEEE Photonics Journal, 4, 1013-1020 (2012).

[15]. Y. Yang, J. Ward, S. Nic Chormaic, "Quasi-droplet microbubbles for high resolution sensing applications," Opt. Express, 22, 6681-6698 (2014). 


\section{Figure Captions:}

Fig. 1 (a) Lead-silicate capillary with an outer diameter of $836.3 \mu \mathrm{m}$ and an inner diameter of $603.5 \mu \mathrm{m}$ measured using an optical microscope; (b) single-stem lead-silicate glass microbubble; (c) Two-stem lead-silicate glass microbubble fabricated.

Fig. 2 Single-stem (left) and two-stem (right) microbubble with a diameter of $38 \mu \mathrm{m}$ and $48 \mu \mathrm{m}$ respectively, both coupled with a tapered silica microfiber with a diameter of circa $1 \mu \mathrm{m}$.

Fig. 3 Resonance spectra of microbubbles with a diameter of (a) $38 \mu \mathrm{m}$ (single-stem) and (b) $48 \mu \mathrm{m}$ (two-stem). One period of the ramp is $80 \mathrm{GHz}$ and the scan frequency is $5 \mathrm{~Hz}$. From the figures, one can see that due to the high contrast of refractive indices between the microbubble and the silica microfiber, this leads to a quasi-periodic spectrum of whispering gallery mode resonances over the entire scanning time.

Fig. 4 FEM simulation of WGM electric field distribution in a lead-silicate microbubble resonator. Left and right are the $n=1, n=2$ and $n=3$ TE modes respectively. The horizontal axis is the radial direction and the vertical access is the polar direction. The outer diameter is $48 \mu \mathrm{m}$ and the wall thickness is $1.2 \mu \mathrm{m}$.

Fig. 5 Effective index of a $48 \mu \mathrm{m}$ diameter lead-silicate microbubble (two-stem sample) for different wall thickness and different modes. Black squares are the fundamental TE mode and red circles are the $2^{\text {nd }}$ order TE mode for a wall thickness from $500 \mathrm{~nm}$, where the air-filled microbubble starts to support high order modes. The effective index of the third radial order is plotted in blue triangles. The taper effective index for a fiber waist of 0.5-2.0 $\mu \mathrm{m}$ diameter is also presented (dashed purple line). Once the geometry of a microbubble is set, a proper taper size can be chosen to satisfy the desired phase matching condition between the microbubble and the tapered silica microfiber. 


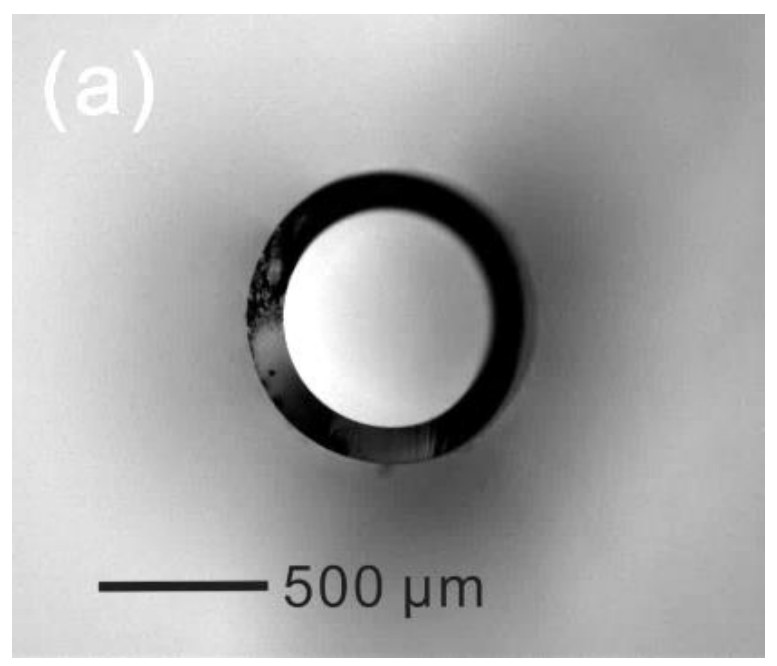

Fig. 1 (a) 


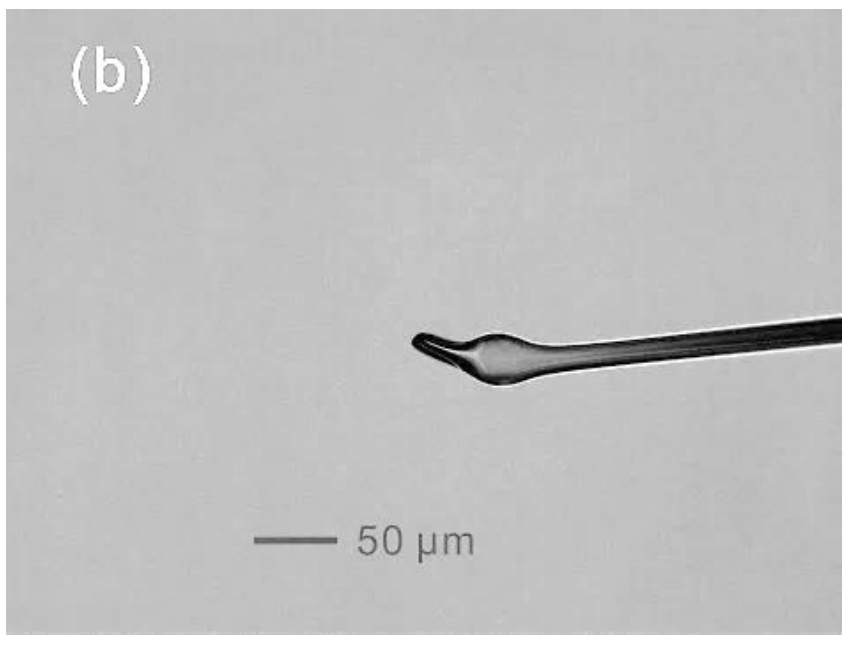

Fig. 1(b) 


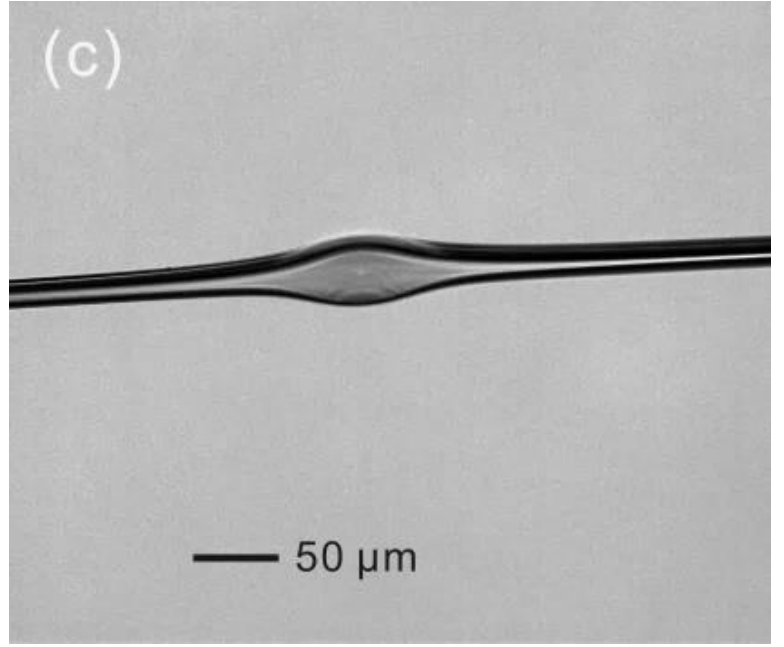

Fig. 1(c) 


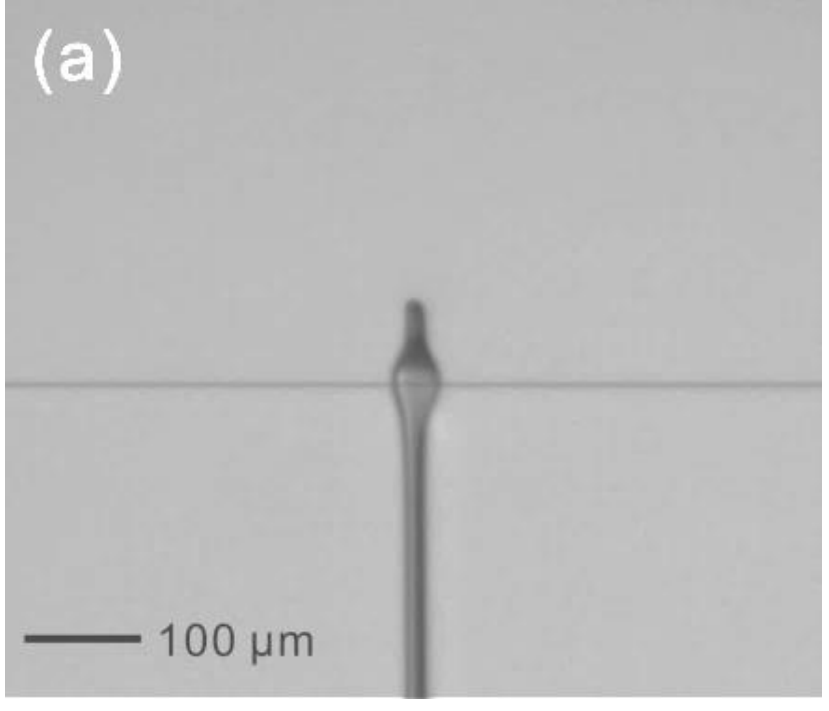

Fig. 2(a) 


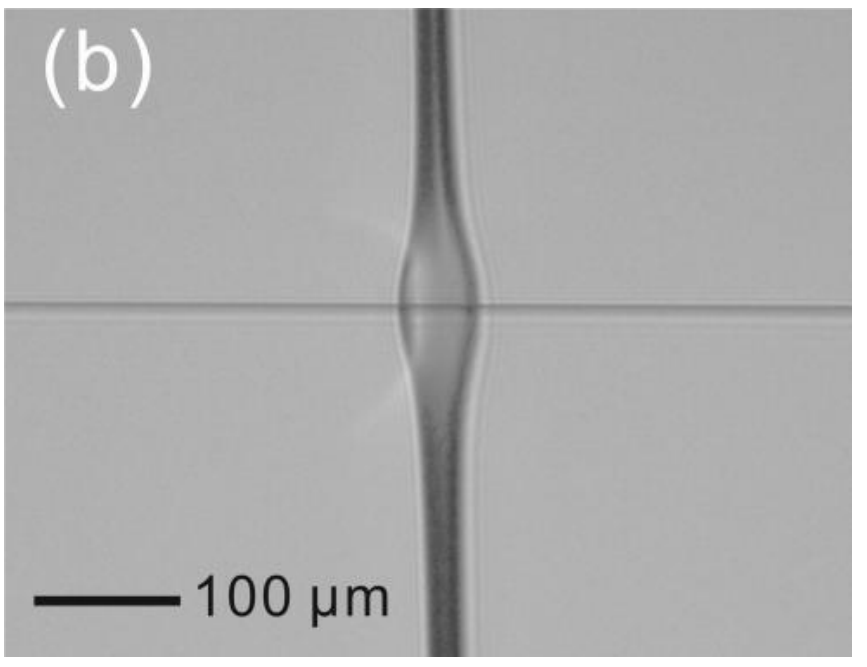

Fig. 2(b) 


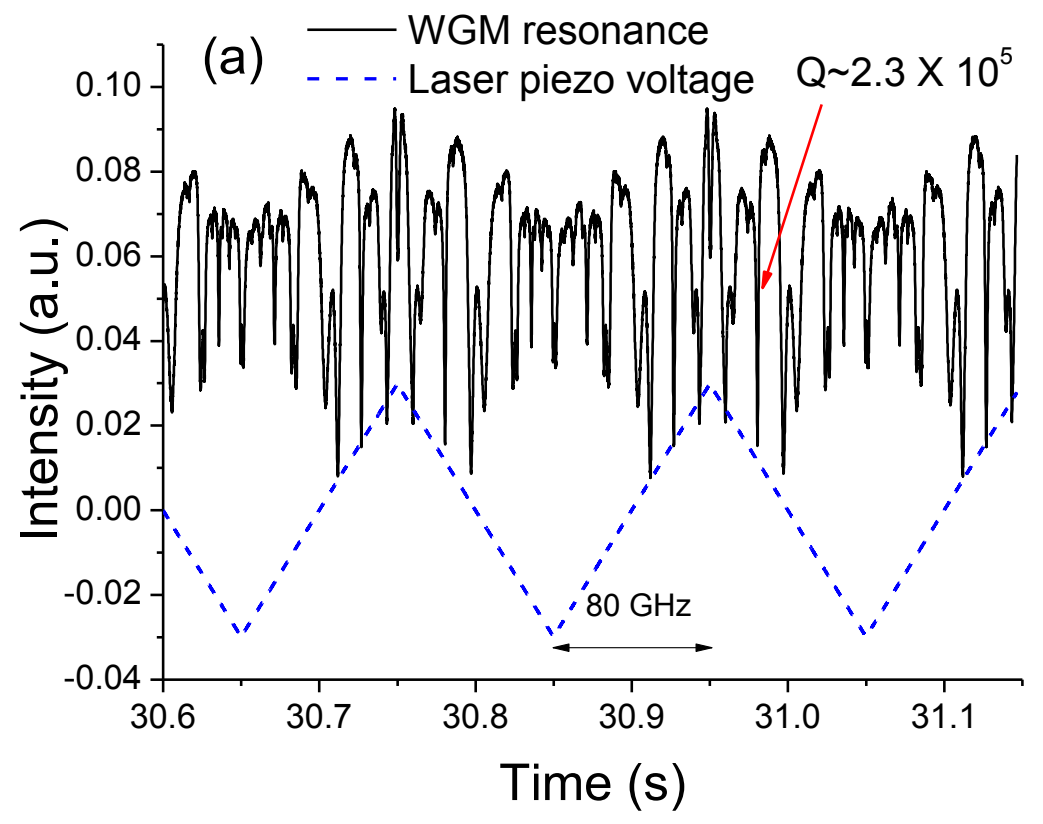

Fig. 3(a) 


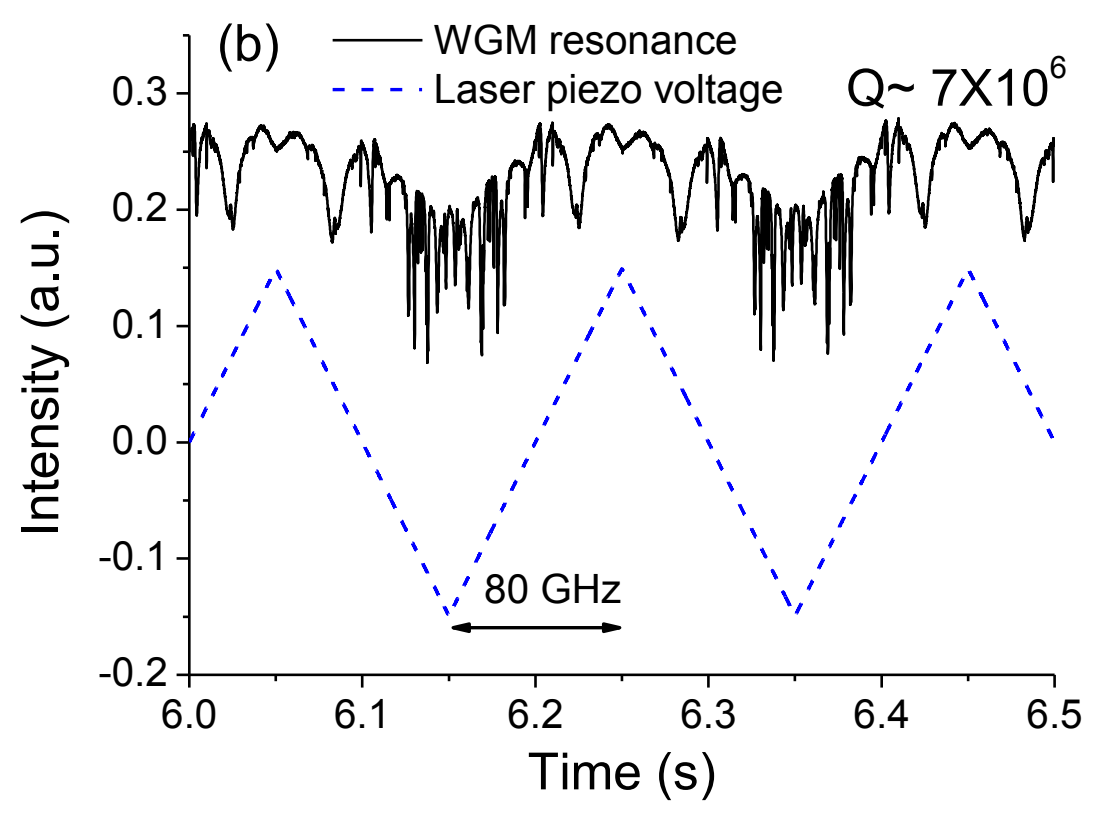

Fig. 3(b) 


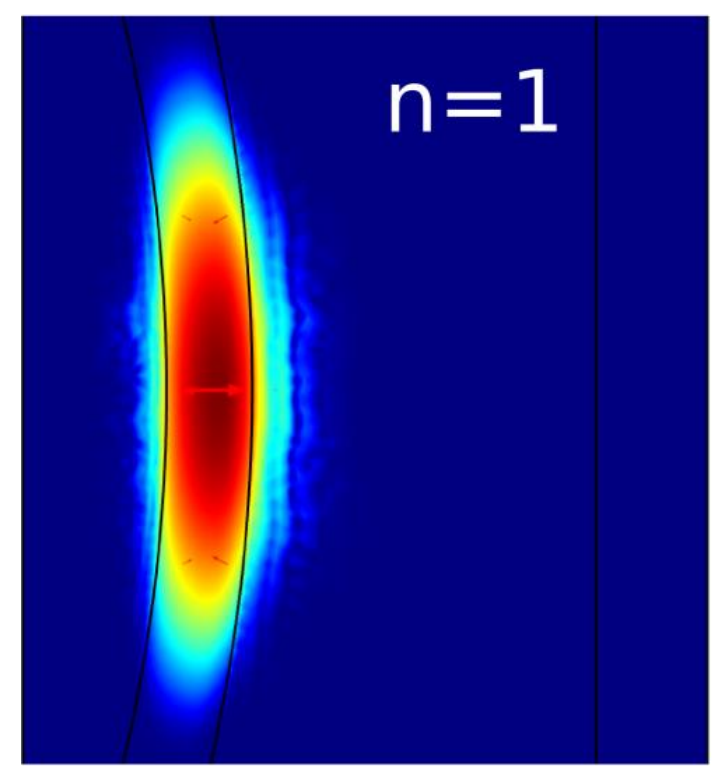

Fig. 4(a) 
$n=2$

Fig. 4(b) 


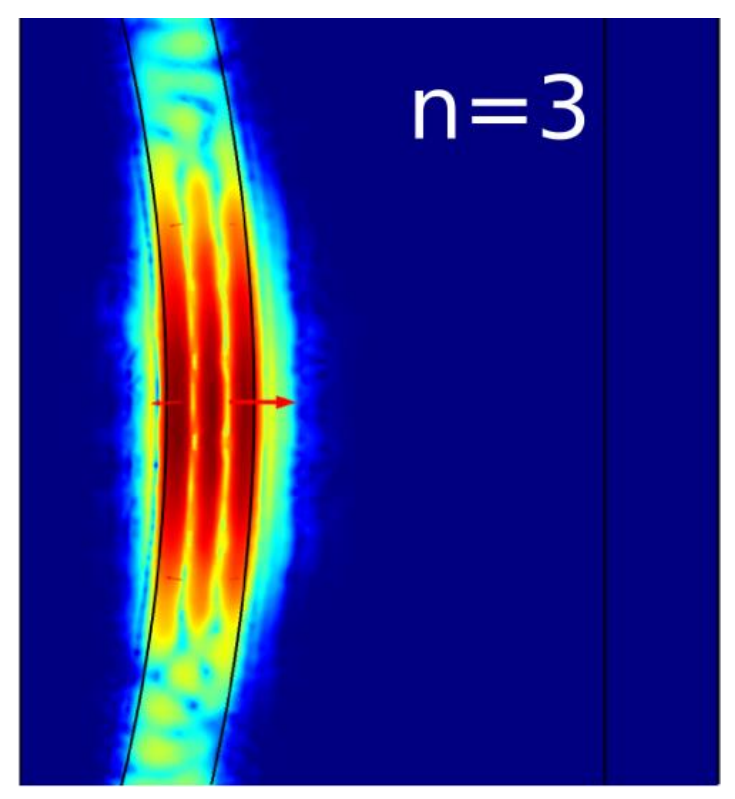

Fig. 4(c) 


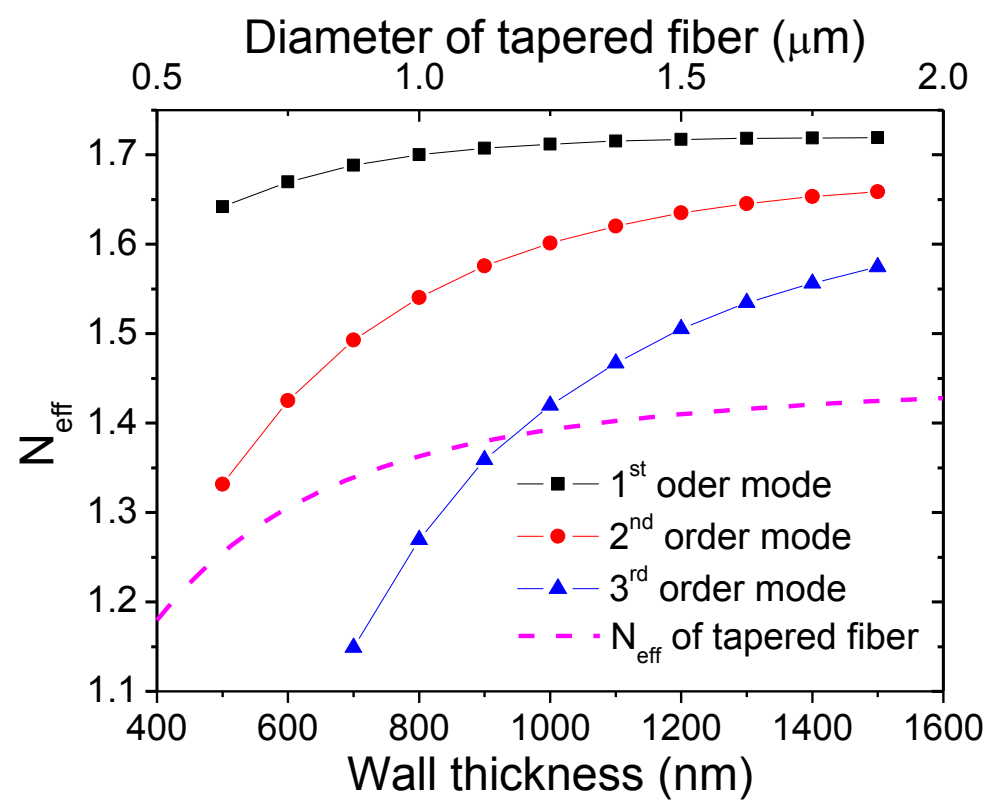

Fig. 5 